\title{
Editorial
}

\section{Biologically Inspired Robotics}

\author{
Liwei Shi, ${ }^{1}$ Maki K. Habib, ${ }^{2}$ Nan Xiao, ${ }^{1,3}$ and Huosheng $\mathrm{Hu}^{4}$ \\ ${ }^{1}$ The Institute of Advanced Biomedical Engineering System, School of Life Science, Beijing Institute of Technology, \\ No. 5, Zhongguancun South Street, Haidian District, Beijing 100081, China \\ ${ }^{2}$ Mechanical Engineering Department, School of Sciences and Engineering, The American University in Cairo, Cairo 11511, Egypt \\ ${ }^{3}$ Faculty of Engineering, Kagawa University, 2217-20 Hayashi-cho, Takamatsu 761-0396, Japan \\ ${ }^{4}$ School of Computer Science and Electronic Engineering, University of Essex, Wivenhoe Park, Colchester CO4 3SQ, UK \\ Correspondence should be addressed to Liwei Shi; shiliwei@bit.edu.cn
}

Received 13 September 2015; Accepted 14 September 2015

Copyright ( 2015 Liwei Shi et al. This is an open access article distributed under the Creative Commons Attribution License, which permits unrestricted use, distribution, and reproduction in any medium, provided the original work is properly cited.

Biologically inspired robotics has enabled today's robots to operate in a variety of unstructured and dynamically changing environments in addition to traditional structured environments. As a result, intelligent robots will soon be ready to serve in our home, hospital, office, and outdoors. It is clear that bioinspired methods are becoming increasingly important in the face of the complexity of today's demanding applications. Biological inspiration in robotics is leading to complex structures with sensory-motor coordination, in which learning often plays an important role in achieving adaptation. This special issue is focused on the theoretical and technological challenges of evolutionary transformation from biological systems to intelligent robots.

The paper "Development and Evaluation of Compact Robot Imitating a Hermit Crab for Inspecting the Outer Surface of Pipes" by N. Imajo et al. concentrates on an outerpipe inspection robot. The robot's locomotion mechanism is developed to imitate a terrestrial hermit crab's claws. It is equipped with two rimless wheels. Each of the spokes is tipped with a neodymium magnet, which allows the robot to remain attached to even a vertical steel pipe. Moreover, the robot has a mechanism for adjusting the camber angle of the right and left wheels, allowing it to tightly grip pipes with different diameters.

The paper "Bioinspired Tracking Control of High Speed Nonholonomic Ground Vehicles" by A. Shoemaker and A. Leonessa presents a control algorithm based on a unicycle model. This algorithm focuses on driving the current vehicle to within some variable following distance of a user defined reference system by commanding longitudinal and angular rates. This algorithm is proven stable through Lyapunov criteria. The results show that the algorithm demonstrates significant promise in high speed trajectory tracking with obstacle avoidance.

The paper "Unified Switching between Flying and Perching of a Bioinspired Robot Using Impedance Control" by S. $\mathrm{Du}$ et al. introduces a unified impedance control approach for bioinspired flying and perching robots. The impedance force control method is developed to enable the robot to approach a desired position and handle the contact force with the environment smoothly until the adsorption device perches on the object surface steadily.

The paper "Rolling Locomotion Control of a Biologically Inspired Quadruped Robot Based on Energy Compensation" by T. Nemoto et al. proposes an approach to control rolling locomotion with the quadruped robot. A dynamic model of the quadruped robot with rolling locomotion is developed by applying a constraint force due to collision and contact with the ground to a base quadruped robot model. The proposed control approach is effective in achieving periodic rolling locomotion.

The paper "Development and Motion Testing of a Robotic Ray" by J. He and Y. Zhang develops a robotic ray based on the simplified pectoral structure of living bluespotted ray. The mechanical structure and control circuit are presented, and kinematic analysis on the fin ray and the full fin is discussed. The average propulsion velocity generally increases with the increase of kinematic parameters such as frequency, amplitude, and wavelength, respectively. 
The paper "Unmanned Aerial Vehicle Navigation Using Wide-Field Optical Flow and Inertial Sensors" by M. B. Rhudy et al. investigates vision-aided inertial navigation techniques which do not rely upon GPS using UAV (unmanned aerial vehicle) flight data. Two different formulations are presented, a full state estimation formulation which captures the aircraft ground velocity vector and attitude and a simplified formulation which assumes that all of the aircraft velocity is in the forward direction. In addition, a state is included in each formulation in order to estimate the distance between the image center and the aircraft.

The paper "Sparse Approximation for Nonrigid Structure from Motion" by Y. Wang et al. proposes a novel sparse approximation method to resolve the nonrigid structure from motion problem in trajectory space. Instead of generating a truncated traditional trajectory basis, this method uses an atom dictionary which includes a set of overcomplete bases to estimate the real shape of the deformable object.

The paper "Simulation of Octopus Arm Based on Coupled CPGs" by J. Tian and Q. Lu presents three coupled central pattern generators (CPGs) and a 2-dimensional dynamic model of the octopus arm to explore possible strategies of the octopus movement control. The CPGs' signals treated as activation are added on the ventral, dorsal, and transversal sides, respectively. The effects of the octopus arm are discussed when the parameters of the CPGs are changed. The results are beneficial for researchers to understand the octopus movement further.

The paper "Action Selection and Operant Conditioning: A Neurorobotic Implementation” by A. Cyr and F. Thériault shows an AS (action selection) process made from simple cellular elements. It is based on a CPG and sensory neurons which influence decision neurons in their choice to generate a behavior from the action neurons. The experiments are conducted under a biologically inspired paradigm, specifically with a SNN (spiking neural network) acting as brain controller for virtual and physical robots. The simplicity and the generic aspect of above AS model may provide a fast track solution to build more complex SNN, including multiple actions in different dynamic scenarios.

The paper "Inverse Kinematic Analysis and Evaluation of a Robot for Nondestructive Testing Application" by Z. Lu et al. provides a simple and effective method for the inverse kinematics problem of an industrial robot system. The armwrist separateness method is adopted to solve the inverse kinematics for robots with $6 \mathrm{DOF}$. The proposed method will greatly benefit the development of nondestructive industrial testing.

Liwei Shi

Maki K. Habib

Nan Xiao

Huosheng $\mathrm{Hu}$ 

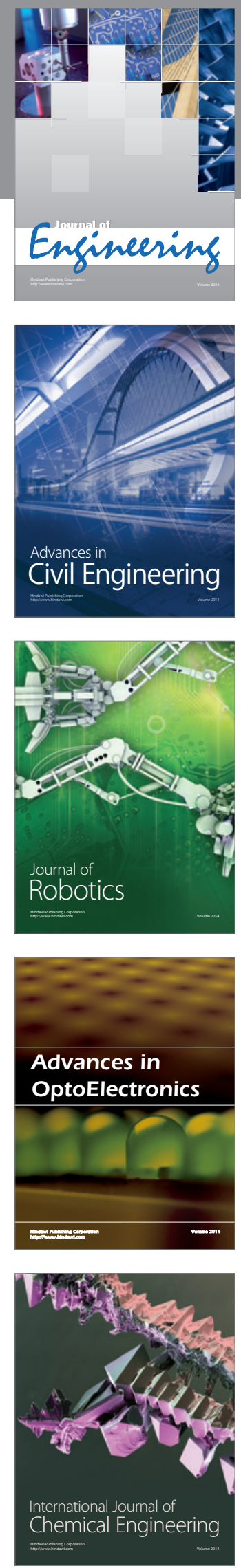

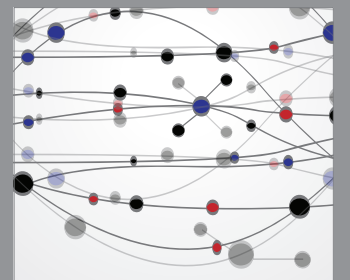

The Scientific World Journal
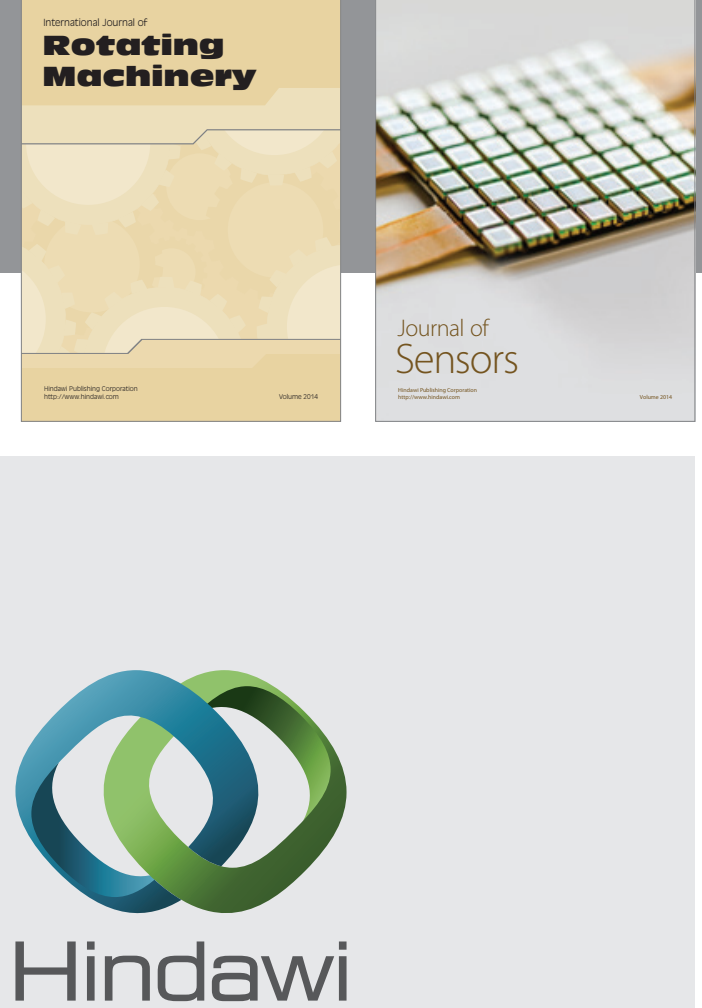

Submit your manuscripts at http://www.hindawi.com
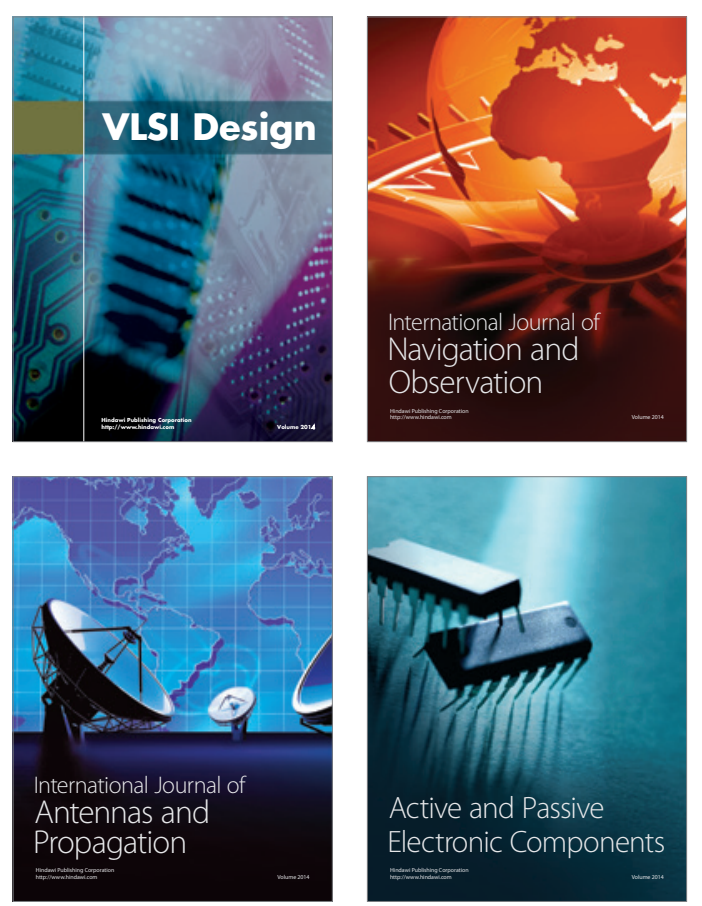
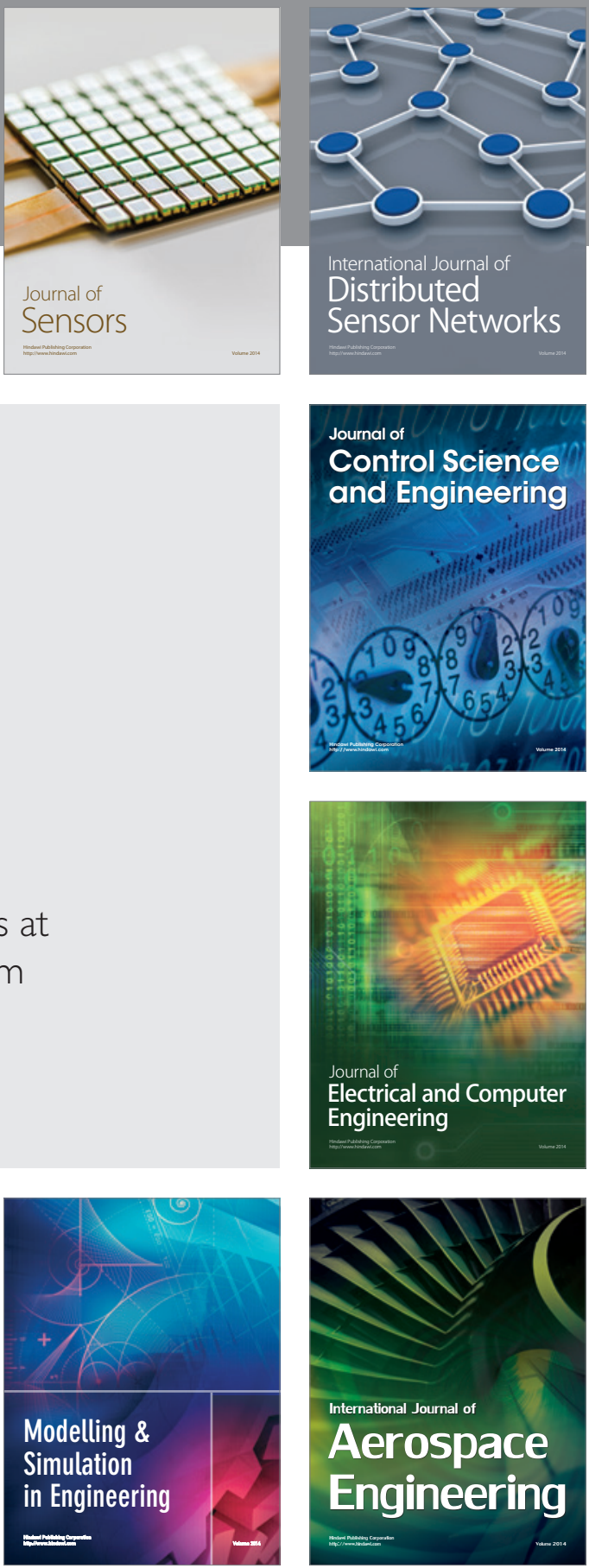

Journal of

Control Science

and Engineering
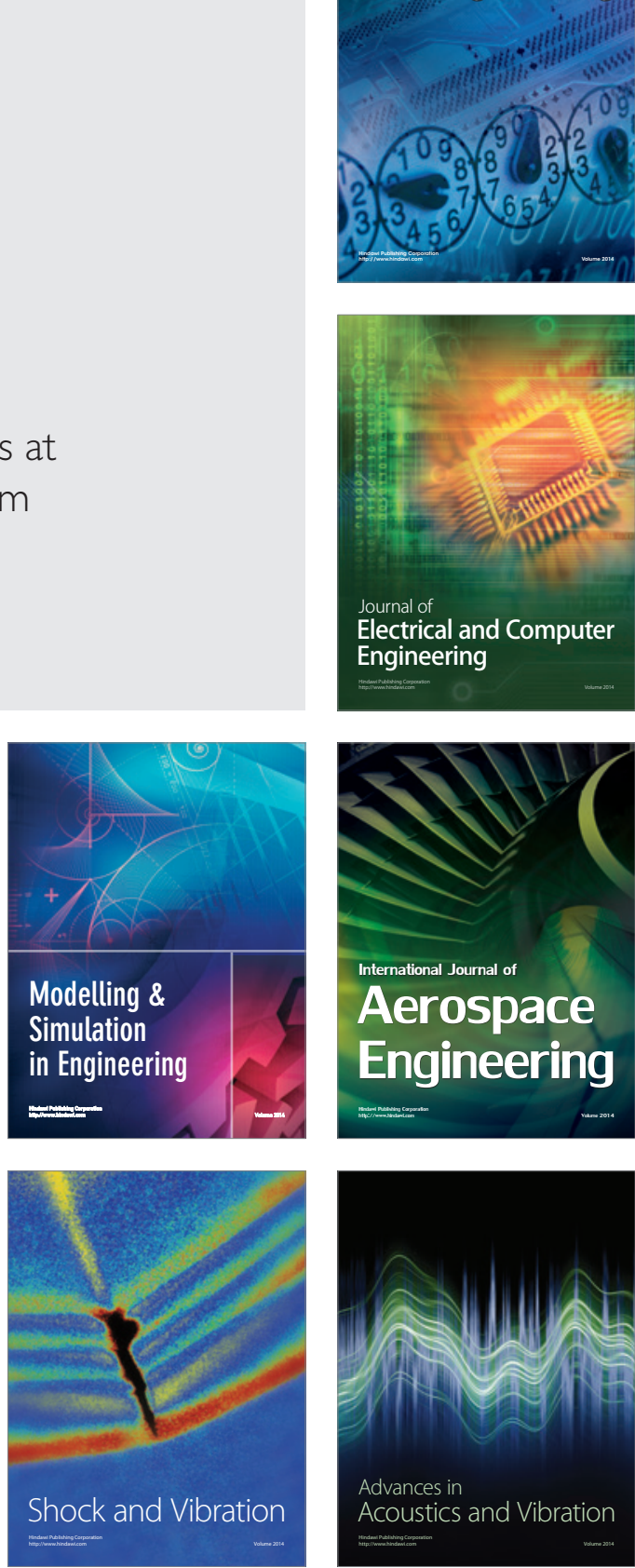